\title{
A New System Identification Approach to Identify Genetic Variants in Sequencing Studies for a Binary Phenotype
}

\author{
Guolian Kang $^{\text {a }}$ Wenjian Bi ${ }^{c}$ Yanlong Zhao ${ }^{c}$ Ji-Feng Zhang ${ }^{c}$ Jun J. Yang ${ }^{b}$ \\ Heng $\mathrm{Xu}^{\mathrm{b}}$ Mignon L. Loh ${ }^{d}$ Stephen P. Hunger ${ }^{\mathrm{e}}$ Mary V. Relling ${ }^{\mathrm{b}}$ \\ Stanley Pounds ${ }^{\mathrm{a}}$ Cheng Cheng ${ }^{\mathrm{a}}$
}

Departments of a Biostatistics and b Pharmaceutical Sciences, St. Jude Children's Research Hospital, Memphis, Tenn., USA; ' Key Laboratory of Systems and Control, Academy of Mathematics and Systems Science, Chinese Academy of Sciences, Beijing, China; 'Benioff Children's Hospital, University of California at San Francisco, San Francisco, Calif., and 'University of Colorado School of Medicine and Children's Hospital Colorado, Aurora, Colo., USA

\section{Key Words}

Set-valued system model · Binary phenotype · Threshold model · Genetic variants · Rare variants · Next-generation sequencing studies

\begin{abstract}
We propose in this paper a set-valued (SV) system model, which is a generalized form of logistic (LG) and Probit (Probit) regression, to be considered as a method for discovering genetic variants, especially rare genetic variants in next-generation sequencing studies, for a binary phenotype. We propose a new SV system identification method to estimate all underlying key system parameters for the Probit model and compare it with the LG model in the setting of genetic association studies. Across an extensive series of simulation studies, the Probit method maintained type I error control and had similar or greater power than the LG method, which is robust to different distributions of noise: logistic, normal, or
\end{abstract}

t distributions. Additionally, the Probit association parameter estimate was 2.7-46.8-fold less variable than the LG logodds ratio association parameter estimate. Less variability in the association parameter estimate translates to greater power and robustness across the spectrum of minor allele frequencies (MAFs), and these advantages are the most pronounced for rare variants. For instance, in a simulation that generated data from an additive logistic model with an odds ratio of 7.4 for a rare single nucleotide polymorphism with a MAF of 0.005 and a sample size of 2,300, the Probit method had $60 \%$ power whereas the LG method had $25 \%$ power at the $a=10^{-6}$ level. Consistent with these simulation results, the set of variants identified by the LG method was a subset of those identified by the Probit method in two example analyses. Thus, we suggest the Probit method may be a competitive alternative to the LG method in genetic association studies such as candidate gene, genome-wide, or next-generation sequencing studies for a binary phenotype.

(C) 2014 S. Karger AG, Base
G. Kang and W. Bi contributed equally to this work.

\section{KARGER}

E-Mail karger@karger.com

www.karger.com/hhe
(C) 2014 S. Karger AG, Basel

0001-5652/14/0782-0104\$39.50/0
Guolian Kang, PhD

Department of Biostatistics, St. Jude Children's Research Hospital

Memphis, TN 38105 (USA)

E-Mail Guolian.Kang@ @ stjude.org

Yanlong Zhao, $\mathrm{PhD}$

Key Laboratory of Systems and Control, Academy of Mathematics and Systems Science Chinese Academy of Sciences

Beijing 100190 (PR China)

E-Mail ylzhao@amss.ac.cn 


\section{Introduction}

For the past 10 years, genome-wide association studies (GWAS) have been an effective and successful method to detect common genetic variations associated with various phenotypes [1-3]. To date, the majority of phenotypes studied have been binary/categorical, continuous, or survival phenotypes. The standard linear regression model is the main method to analyze continuous phenotypes if the normality assumption holds approximately for its original or transformed scale. The Cox proportional hazard regression model is the key method to analyze the survival outcomes if the proportional hazard assumption holds approximately. The logistic (LG) regression model is widely used to analyze the binary/categorical phenotype in GWAS. Often, a binary phenotype is derived from a continuous variable by splitting the range at some threshold and categorizing individuals above and below that point into 2 separate groups of 'affected' and 'unaffected'. Examples of such designations include obesity defined based on body mass index [4], hypertension defined based on systolic and/or diastolic blood pressure [5], and diabetes defined based on hemoglobin A1c level [6]. Moreover, some binary phenotypes may manifest from complicated unobserved or unobservable continuous variables such as expression of an unknown protein in a particular organ that causes the disease. Therefore, the simple LG model may be too naive to adequately reflect the underlying biology, resulting in performance reduction in studies of phenotypes as such.

The Cochran-Armitage trend test (CATT) is a widely used test for the binary phenotype (case-control) which assumes an additive mode of inheritance. CATT is equivalent to the score test for a logistic regression and has high power for additive and multiplicative disease models but much lower power for the recessive disease model [7-10]. The genotypic association test using Pearson's $\chi^{2}$ test for a $2 \times 3$ contingency table is robust for different disease models [11] but generally has lower power than CATT for the additive disease model. MAX3 is another widely used method that is robust for different disease models [12]. MAX3 is the maximum of the absolute values of CATT statistics, assuming the additive, dominant, and recessive disease modes of inheritance. The p value of MAX3 can be estimated by the approximation method implemented in the software [13] because of its complicated asymptotic distribution. Other innovative methods include the entropy-based method which is generally as good as or more powerful than the genotypic association test [11, 14], and some genetic model selection and genetic model

The Probit Regression to Test Genotype/

Binary Phenotype Associations exclusion methods based on Hardy-Weinberg disequilibrium information [15-17]. Although these methods have some advantages in certain situations, they either cannot adjust for confounding factors such as genetic ancestry, which is commonly adjusted in genetic association studies, as the LG model does, are time consuming when applied to GWAS, or can have lower power than CATT when the underlying disease model is additive or multiplicative [18], especially for a small sample size.

The set-valued system widely exists in reality. And in some cases, it can degenerate to the well-known threshold model. The corresponding set-valued system identification was first investigated for sensor systems [19]. In contrast to the traditional system identification method, setvalued system identification can estimate the model by set-valued information rather than the precise output. Although set-valued system identification is technically challenging, it has been successfully used in various fields such as sensor networks and telecommunications [20, $21]$. If the outcome is a linear function of covariates and the noise follows a normal distribution, then the set-valued system model is referred to as the Probit model, which is a viable choice for genetic association studies. If the noise follows a logistic distribution, then the set-valued system model is referred to as the logistic model, which is widely used in genetic association studies.

While it is widely believed that LG and Probit give very similar statistical analysis results in most applications because the cumulative distribution function of the standard normal distribution is very similar to that of the logistic distribution with a mean of 0 and a scale of 1 [22], some published research suggests that there may be some GWAS applications in which the two models have very different statistical properties. The LG model can be poorer than the Probit model in terms of goodness of fit in small sample size settings because the logistic distribution has heavier tails than the normal distribution [23]. Also, linear discriminate analysis, which like Probit regression is based on a normal distribution instead of a logistic distribution, has much greater asymptotic efficiency than LG regression [24]. Furthermore, it has been shown that a toxicology study that evaluates a binary response at 3 drug dose levels $(-0.79,+0.79$, and +2.69 on an arbitrary scale) with most subjects assigned to one of the extreme doses is optimal for differentiating between a LG and Probit regression model in terms of goodness of fit [25]. Furthermore, since changes of \pm 0.2 in the dose levels have minimal impact on the power to distinguish between the LG and Probit models [24] and the statistical results are invariant to shift and scale transformations of 
the dose levels, the result holds for transformed dose levels 0,1 , and 2 , which is equivalent to the common representation of genotype data as the number of copies of the minor alleles. This result suggests that the LG and Probit models may have very different performance in terms of type I error control and power in the analysis of the association of rare variants with disease status.

In this study, we propose a set-valued (SV) system model, which considers the dichotomization process of continuous phenotypes to model the relationship between the binary outcome and possible genetic or nongenetic explanatory factors in GWAS or next-generation sequencing (NGS) studies. We propose a set-valued system identification approach for the Probit model to estimate the parameter of interest and use a Wald test statistic for testing the null hypothesis $\left(H_{0}\right)$ of no genotype/ binary phenotype association. We performed extensive simulation studies to compare the type I error rate and power of the Probit and LG methods. Finally, we applied both methods to a mini-exome sequencing data set and a candidate gene study.

\section{Materials and Methods}

\section{Notation}

We assume that there are $N_{0}$ cases and $N_{1}$ controls in a casecontrol genetic association study (total sample size $N=N_{0}+N_{1}$ ) and that the genetic polymorphism of interest is diallelic (e.g. a single nucleotide polymorphism; SNP). The 2 alleles at a SNP are denoted as $\mathrm{A}$ and $\mathrm{a}$, where $\mathrm{A}$ is the minor allele. The 3 genotypes are therefore AA, Aa, and aa. Suppose that observations $\left(s_{i}, X_{i}, G_{i}\right)$ are available for $N$ individuals, $i=1,2, \ldots, N$, where $s_{i}$ is the casecontrol status of subject $i, X_{i}=\left[x_{i 1}, x_{i 2}, \ldots, x_{i m}\right]^{\mathrm{T}}$ is the vector of $m$ covariates that we need to adjust for (e.g. demographic or clinical variables), and $G_{i}=0,1$, or 2 is the numerical coding of the 3 genotypes aa, Aa and AA of the SNP for the same individual.

\section{LG Model}

The LG model [26] commonly used to test the association between a SNP and a binary phenotype with adjustment for some covariates is

$$
\text { logit } \operatorname{Pr}\left(s_{i}=1\right)=\alpha_{0}+\theta G_{i}+\gamma^{T} X_{i}, i=1,2, \ldots, N,
$$

where $\alpha_{0}$ is an intercept term, $\theta$ is the regression coefficient for the SNP and $\gamma=\left[\gamma_{1}, \gamma_{2}, \ldots, \gamma_{m}\right]^{T}$ is a vector of regression coefficients for $m$ covariates. The above equation is equivalent to

$$
\operatorname{Pr}(s=1)=\frac{1}{1+\mathrm{e}^{-\left(\alpha_{0}+\theta G_{i}+\gamma^{T} X_{i}\right)}} .
$$

Evaluating whether the genetic variant SNP influences the phenotype, adjusting for covariates, corresponds to testing $H_{0}: \theta=0$. The Wald test will be used to test for $H_{0}$ in order to be consistent with the Probit method below.

\section{SV Model}

Instead of directly modeling the relationship between the genetic variant and the phenotype using the LG model, we propose a new SV system model in which the observation of cases and controls are measured by a set-valued sensor $[20,21]$

$$
\left\{\begin{array}{l}
y=f(G, X)+e, \\
s=I(y \in A),
\end{array}\right.
$$

where $I(y \in A)$ is the indicate function of set $A, f$ is a deterministic function of $G$ and $X, y$ is a latent continuous variable, and $e$ is the random noise. The most common simplified case of set-valued sensor is to introduce a threshold $c$ to dichotomize the continuous variable. In this case, the SV model is very similar to the wellknown threshold model

$$
\left\{\begin{array}{l}
y=f(G, X)+e \\
s=I(y>c) .
\end{array}\right.
$$

Furthermore, when function $f$ is a linear function of $G$ and $X$ and $e$ follows a normal distribution, the SV model becomes the Probit model [26]

$$
\left\{\begin{array}{l}
y_{i}=\alpha_{0}+\theta G_{i}+\gamma^{T} X_{i}+e_{i}, \\
s_{i}=I\left(y_{i}>c\right), i=1,2, \ldots, N,
\end{array}\right.
$$

where $y_{i}$ is a latent continuous variable that can be dichotomized as case/control, $e_{i}$ is the independent and identically distributed random noise which follows a normal distribution with a mean of 0 and a variance of $\sigma^{2}, c$ is the threshold used to define case/control status, and the observation $s_{i}$ is determined by a threshold $c$ and the latent variable $y_{i} . H_{0}: \theta=0$ corresponds to no genetic effect of the SNP on the phenotype. The parameter $\theta$ is to be identified to test for $H_{0}$ using the expectation-maximization (EM) algorithm below.

Note if function $f$ is a linear function of $G$ and $X$, but $e$ follows a logistic distribution with a location 0 and a scale of 1 , then the SV model becomes the LG model

$$
\operatorname{Pr}(s=1)=\frac{1}{1+\mathrm{e}^{\tilde{\alpha}+\tilde{\theta} G+\tilde{\gamma}^{T} X}}=\frac{1}{1+\mathrm{e}^{c-\left(\alpha_{0}+\theta G_{i}+\gamma^{T} X_{i}\right)}},
$$

if $c=0$, then it becomes the LG model (see equ. 1 ).

Similarly, it can be seen that

$$
\operatorname{Pr}(s=1)=\Phi\left(\tilde{\alpha}+\tilde{\theta} G+\tilde{\gamma}^{T} X\right),
$$

where $\Phi$ is the cumulative distribution function for standard normal distribution,

$$
\tilde{\alpha}=-\frac{c-\alpha_{0}}{\sigma}, \tilde{\theta}=\frac{\theta}{\sigma}, \tilde{\gamma}=\frac{\gamma}{\sigma} .
$$

However, an important deviation from the usual Probit model is that here we take a novel system identification approach to estimate all key underlying system parameters $\theta, \gamma$, and $c$ (see below). We call equation 2 the Probit model but coupled with the new algorithm in the remaining of the paper. The core algorithm of the system identification is the EM algorithm, instead of the traditional Newton-like method which is widely used in the usual Probit regression method. The EM algorithm has outstanding robustness and, without calculating the Hessian-like matrix for every iteration 
step, it takes much less time per iteration. Hence, we expect that this approach will be more efficient when the binary observations approximately follow the model in equation 2 . The details about the algorithm and the efficiency discussions can be seen in the Discussion section, and see online supplementary section 1 and supplementary table S2 (for all online suppl. material, see www. karger.com/doi/10.1159/000363660).

\section{Estimation of $\theta$ and the Test Statistic}

The system parameters in equation 1 can be estimated by maximum likelihood through the EM algorithm [21]. Denote the vector of parameters $\theta, \gamma^{T}$, and $c$ by $\Theta$, the vector of $G, X$, and -1 by $\varphi$, and the maximum likelihood estimator of $\Theta$ by $\hat{\Theta}$. The iteration process of the EM-based system identification and the Fisher information matrix of $\Theta$ at $\hat{\Theta}$, denoted as $i(\hat{\Theta})$, can be obtained as

$$
\hat{\Theta}^{k+1}=\hat{\Theta}^{k}+\left(\sum_{i=1}^{N} \phi_{i} \cdot \phi_{i}^{T}\right)^{-1}\left[\sum_{i=1}^{N} f\left(\hat{\alpha}_{i}^{k}\right)\left(\frac{I\left(s_{i}=1\right)}{1-F\left(\hat{\alpha}_{i}^{k}\right)}-\frac{I\left(s_{i}=0\right)}{F\left(\hat{\alpha}_{i}^{k}\right)}\right) \cdot \sigma^{2} \phi_{i}\right]
$$

(for details, see online suppl. section 1) and

$$
\mathrm{i}(\hat{\Theta})=-E\left[\frac{\partial^{2}}{\partial \Theta^{2}} \log L(\Theta) \mid \hat{\Theta}\right]=\sum_{\mathrm{i}=1}^{N} \frac{1}{F(1-F)} f^{2} \cdot \phi_{i} \cdot \phi_{i}^{T},
$$

where $L(\Theta)$ is the likelihood function and $F($.$) and f($.$) are the cu-$ mulative distribution function and probability distribution function of a normal distribution with a mean of 0 and a variance of $\sigma^{2}$, respectively. Testing for no genetic effect of the SNP on the phenotype, i.e. $H_{0}: \theta=0$, can be constructed for the Probit method from the Wald statistic

$$
W=\frac{\hat{\theta}^{2}}{\mathrm{i}(\hat{\Theta})^{-1}[1,1]},
$$

where $i(\hat{\Theta})^{-1}[1,1]$ is the variance of $\hat{\theta}$, i.e. the element at the first row and column of the inverse Fisher information matrix. Asymptotically, for large sample sizes, $W$ is distributed approximately as a central $\chi^{2}$ distribution with 1 degree of freedom under $H_{0}$ of no association between the SNP and the phenotype.

\section{Simulations}

\section{Data Generation}

Simulation studies were performed to compare the relative performance of the Probit method coupled with the proposed EM algorithm and the LG method. In these simulations, given the minor allele frequency (MAF) $p_{\mathrm{A}}$, the genotype frequencies $p(G=g)$ were calculated according to the Hardy-Weinberg equilibrium (HWE) law, i.e. $p(G=0)=\left(1-p_{A}\right)^{2}, p(G=1)=2 p_{A}\left(1-p_{A}\right), p(G=2)=$ $\left(p_{A}\right)^{2}$. Two covariates were considered: $x_{1}$ was a binary variable that is 1 with a probability of 0.5 and 0 otherwise and $x_{2}$ was a continuous variable that follows a standard normal distribution. The genotypes and 2 covariates for a population of 2,000,000 individuals were independently generated from their respective distributions.

The case-control status was determined from the generated genotype and covariate data according to two models, respectively.

(1) LG model (LGsimu):

$$
\operatorname{Pr}\left(s_{i}=1 \mid G_{i}, x_{i 1}, x_{i 2}\right)=\frac{\exp \left(\alpha_{0}+\theta G_{i}+0.5 x_{i 1}+0.5 x_{i 2}\right)}{1+\exp \left(\alpha_{0}+\theta G_{i}+0.5 x_{i 1}+0.5 x_{i 2}\right)},
$$

The Probit Regression to Test Genotype/

Binary Phenotype Associations where $\alpha_{0}=-2.2$ is the parameter chosen so that the disease prevalence is 0.1 among the subpopulation with $x_{1}=x_{2}=G=0$.

(2) Probit model (PRsimu): first a continuous variable was generated from

$$
y_{i}=\alpha_{0}=\theta G_{i}+0.5 x_{i 1}+0.5 x_{i 2}+e_{i},
$$

where $e_{i}$ follows the standard normal distribution and $\alpha_{0}=-2.2$. Then, the individuals with a large value of $y_{i}$ greater than threshold $c=\Phi^{-1}(1-0.1)+\alpha_{0}$ were declared as cases and the remaining individuals as controls. This model also gives a disease prevalence of 0.1 among the subpopulation with $x_{1}=x_{2}=G=0$.

Then, $n$ cases and $n$ controls were randomly selected from a population of 2,000,000 individuals.

\section{Type I Error Rate Simulations}

Eight values for MAFs of SNPs were considered: 0.005, 0.01, $0.05,0.1,0.2,0.3,0.4$, and 0.5 . The case-control status was determined from the generated genotype and covariate data using the 2 models mentioned above, with $\theta=0$. To estimate the type I error rate of the Probit and LG method, 10,000,000 replicated data sets were simulated for each case-control study, with a small sample size of 500 cases and 500 controls and a large sample size of 2,000 cases and 2,000 controls, for larger significant levels $\alpha=0.05$ or 0.01 , and stringent genome-wide levels $\alpha=10^{-5}$ or $10^{-6}$ under $H_{0}$ : $\theta=0$, respectively.

\section{Power Simulations}

Three genetic disease models were considered: additive, dominant, and recessive. The case-control status was determined from the generated genotype and covariate data according to the simulation methods given above, with $\theta$ varying from 0.3 to 2 at an increment of 0.1 . Data sets were generated 10,000 times for each configuration. The LG and Probit methods used for the type I error simulations were applied to each data set, and power was estimated as the proportion of $\mathrm{p}$ values $<\alpha=10^{-6}$.

\section{Simulation Results}

\section{Type I Error Rate}

Table 1 shows empirical type I error rates estimated for both the LG and Probit methods. Regardless of significance levels, both methods correctly maintained type I error rates at the given levels but both are conservative if the SNPs are rare and the sample size is small because of large variances of parameter estimates (table 2, online suppl. fig. S1).

\section{Power of the LG and Probit Methods}

Figures 1 and 2 show the power of the LG and Probit methods as a function of effect size $(\theta)$, for additive and dominant disease models, for $n=500$ and 1,000. As expected, the power of both methods increased with the increase in effect size. For a common SNP with a MAF of 0.2 or 0.05 , the estimated coefficient of the Probit method 
Table 1. Type I error of the Probit and the LG method

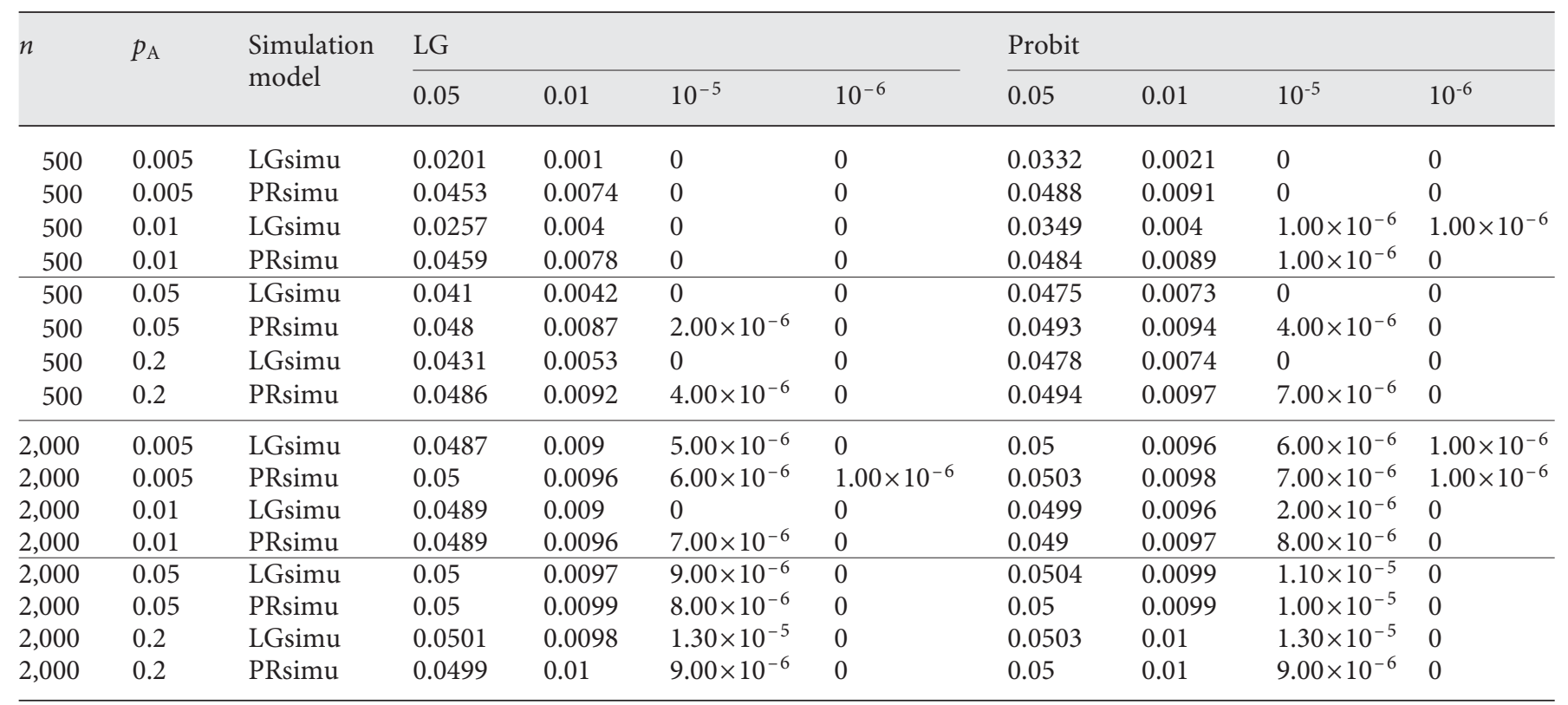

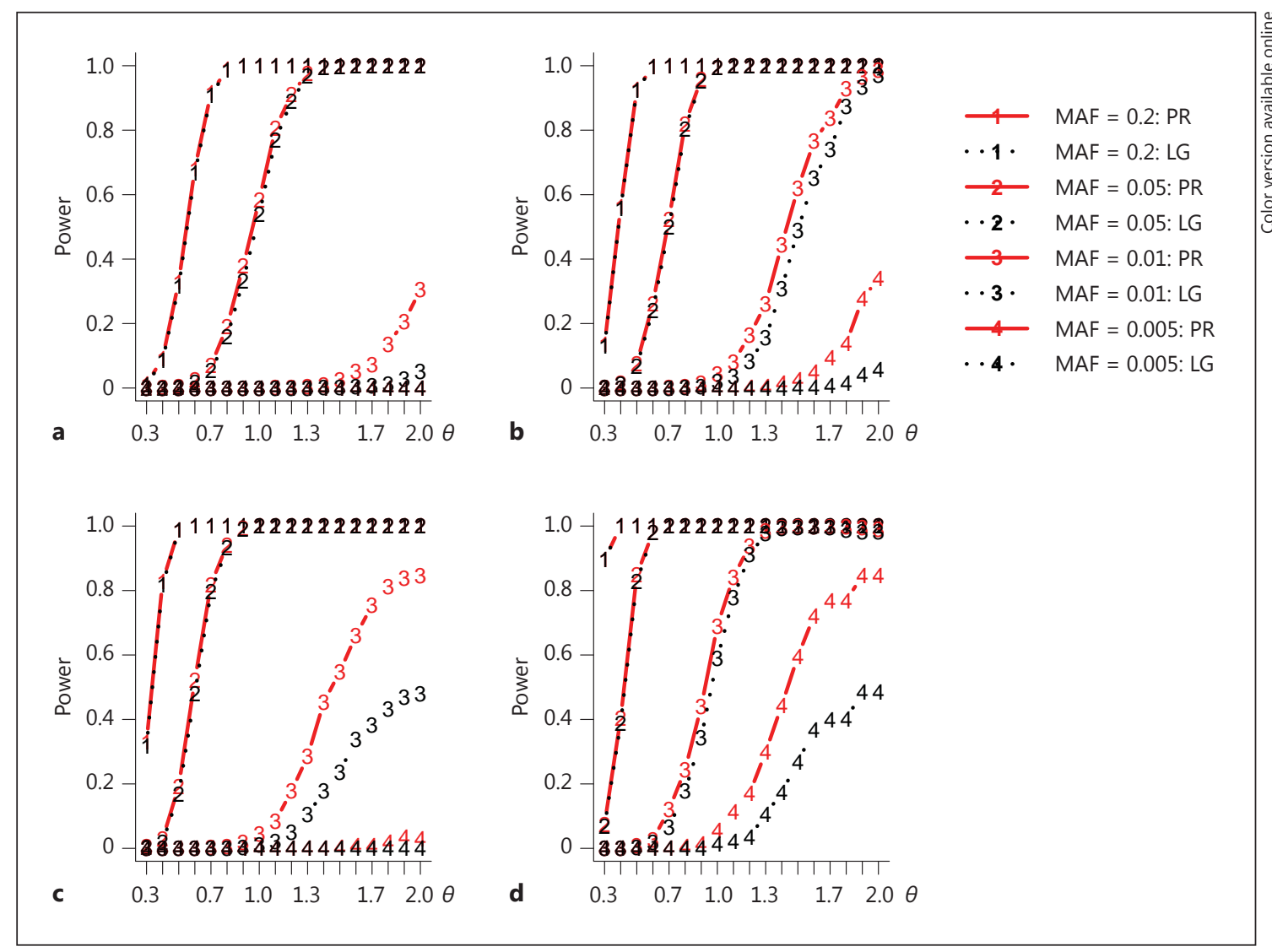

Fig. 1. Power of the Probit (PR) method and the LG method for the additive model using LGsimu (a, b) and PRsimu (c, d). The significance level of the test was $1 \times 10^{-6} \cdot n=500(\mathbf{a}, \mathbf{c}) \cdot n=1,000(\mathbf{b}, \mathbf{d})$. 
Table 2. Comparison of performances of the Probit and the LG method

\begin{tabular}{|c|c|c|c|c|c|c|c|c|c|c|c|c|}
\hline$n$ & $p_{\mathrm{A}}$ & $\theta$ & $\begin{array}{l}\text { Simulation } \\
\text { model }\end{array}$ & $\begin{array}{l}\text { Disease } \\
\text { model }\end{array}$ & \multicolumn{3}{|l|}{ LG } & \multicolumn{3}{|l|}{ Probit } & \multicolumn{2}{|c|}{ LG/Probit } \\
\hline 500 & 0.005 & 0 & LGsimu & $H_{0}$ & -0.1296 & 67.125 & 4.3431 & -0.0456 & 0.8542 & 0.3906 & 2.84 & 11.12 \\
\hline 500 & 0.005 & 0.5 & LGsimu & $\mathrm{ADD}$ & 0.6552 & 24.02 & 2.809 & 0.3473 & 0.4247 & 0.2837 & 1.89 & 9.903 \\
\hline 500 & 0.005 & 2 & LGsimu & $\mathrm{ADD}$ & 2.88 & 589.52 & 11.216 & 1.334 & 0.7324 & 0.3975 & 2.16 & 28.21 \\
\hline 500 & 0.005 & 0.5 & PRsimu & $\mathrm{ADD}$ & 1.077 & 29.91 & 2.7258 & 0.5824 & 0.415 & 0.2645 & 1.85 & 10.31 \\
\hline 500 & 0.005 & 2 & PRsimu & $\mathrm{ADD}$ & 7.263 & 18442 & 39.188 & 2.407 & 2.506 & 0.8591 & 3.02 & 45.61 \\
\hline 500 & 0.2 & 0 & LGsimu & $H_{0}$ & 0.00732 & 0.0135 & 0.0147 & 0.0046 & 0.0052 & 0.0056 & 1.58 & 2.63 \\
\hline 500 & 0.2 & 0.5 & LGsimu & $\mathrm{ADD}$ & 0.5079 & 0.013 & 0.013 & 0.3123 & 0.0048 & 0.0049 & 1.63 & 2.673 \\
\hline 500 & 0.2 & 2 & LGsimu & $\mathrm{ADD}$ & 2.014 & 0.0207 & 0.0211 & 1.206 & 0.0067 & 0.007 & 1.67 & 2.998 \\
\hline 500 & 0.2 & 0 & PRsimu & $H_{0}$ & -0.005 & 0.016 & 0.017 & -0.0026 & 0.0058 & 0.0061 & 1.95 & 2.779 \\
\hline 2,000 & 0.005 & 0.5 & LGsimu & $\mathrm{ADD}$ & 0.5008 & 0.0993 & 0.1023 & 0.3071 & 0.0371 & 0.038 & 1.63 & 2.691 \\
\hline 2,000 & 0.005 & 2 & LGsimu & $\mathrm{ADD}$ & 2.033 & 0.1134 & 0.1128 & 1.199 & 0.0333 & 0.0329 & 1.7 & 3.427 \\
\hline 2,000 & 0.005 & 0 & PRsimu & $H_{0}$ & -0.0177 & 0.1351 & 0.1515 & -0.0108 & 0.0488 & 0.0541 & 1.64 & 2.797 \\
\hline 2,000 & 0.005 & 0.5 & PRsimu & $\mathrm{ADD}$ & 0.916 & 0.1113 & 0.1161 & 0.5485 & 0.0392 & 0.041 & 1.67 & 2.834 \\
\hline 2,000 & 0.005 & 2 & PRsimu & $\mathrm{ADD}$ & 3.649 & 2.1054 & 1.002 & 2.034 & 0.0748 & 0.0832 & 1.79 & 12.05 \\
\hline 2,000 & 0.2 & 0 & LGsimu & $H_{0}$ & 0.00376 & 0.0034 & 0.003 & 0.0024 & 0.0013 & 0.0011 & 1.57 & 2.621 \\
\hline 2,000 & 0.2 & 0.5 & LGsimu & $\mathrm{ADD}$ & 0.503 & 0.0032 & 0.0033 & 0.3094 & 0.0012 & 0.0012 & 1.63 & 2.675 \\
\hline 2,000 & 0.2 & 2 & LGsimu & $\mathrm{ADD}$ & 2.004 & 0.0051 & 0.0053 & 1.201 & 0.0017 & 0.0018 & 1.67 & 2.96 \\
\hline 2,000 & 0.2 & 0 & PRsimu & $H_{0}$ & -0.0091 & 0.004 & 0.004 & -0.0052 & 0.0014 & 0.0014 & 1.76 & 2.767 \\
\hline 2,000 & 0.2 & 0.5 & PRsimu & $\mathrm{ADD}$ & 0.8683 & 0.0039 & 0.0039 & 0.5218 & 0.0014 & 0.0014 & 1.66 & 2.832 \\
\hline 2,000 & 0.2 & 2 & PRsimu & $\mathrm{ADD}$ & 3.425 & 0.0109 & 0.0105 & 1.997 & 0.0031 & 0.0031 & 1.72 & 3.395 \\
\hline
\end{tabular}

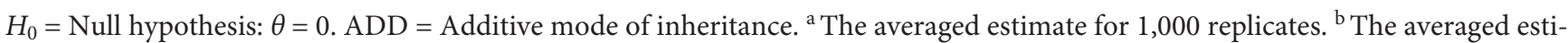
mated asymptotic variance for 1,000 replicates. ${ }^{\mathrm{c}}$ The empirical variance for 1,000 replicates.

was 1.6-1.8 times that of the LG method (table 2), which is the case when both models fit well [22], so it is not surprising that the power of the Probit method was almost identical to or slightly greater than that of the LG method (fig. 1, 2), regardless of the effect sizes of the SNP $(\theta)$ and the genetic disease model. For a rare SNP with a MAF of 0.01 or 0.005 , the power of the Probit method was much greater than that of the LG method, regardless of the genetic disease model. The gain in efficiency for the new Probit method was noticeable in detecting rare variants with moderate sample sizes (fig. 1-3). If the phenotype was simulated using LGsimu under the additive model, with a total sample size of 1,000 , the power of the Probit method was $31 \%$, whereas that of the LG method was $0.05 \%$ for detecting a rare SNP with a MAF of 0.01 and an effect size of 2 (fig. 1b). For a total sample size of 2,000, the power of the Probit method was $34 \%$, whereas that of the LG method was only $6 \%$ for detecting a rare SNP with a MAF of 0.005 and an effect size of 2 (fig. 1d). If the phenotype was simulated using PRsimu under the additive model, with a total sample size of 1,000 , the power of the Probit method was $81 \%$, whereas that of the LG method was $43 \%$ for detecting a rare SNP with a MAF of 0.01 and an effect size of 1.8 (fig. 2b). For a total sample size of 2,000 , the power of the Probit method was $77 \%$, whereas that of the LG method was only $39 \%$ for detecting a rare SNP with a MAF of 0.005 and an effect size of 1.8 (fig. 2d).

Figure 3 displays the power of the LG and Probit methods as a function of sample size for the additive and dominant disease models. As expected, the power of both the LG and Probit methods increased with an increase in sample size. For a common SNP with a MAF of 0.2 or 0.05 and an effect size of 0.4 or 0.8 , respectively, the power of the Probit method was almost identical to that of the LG 


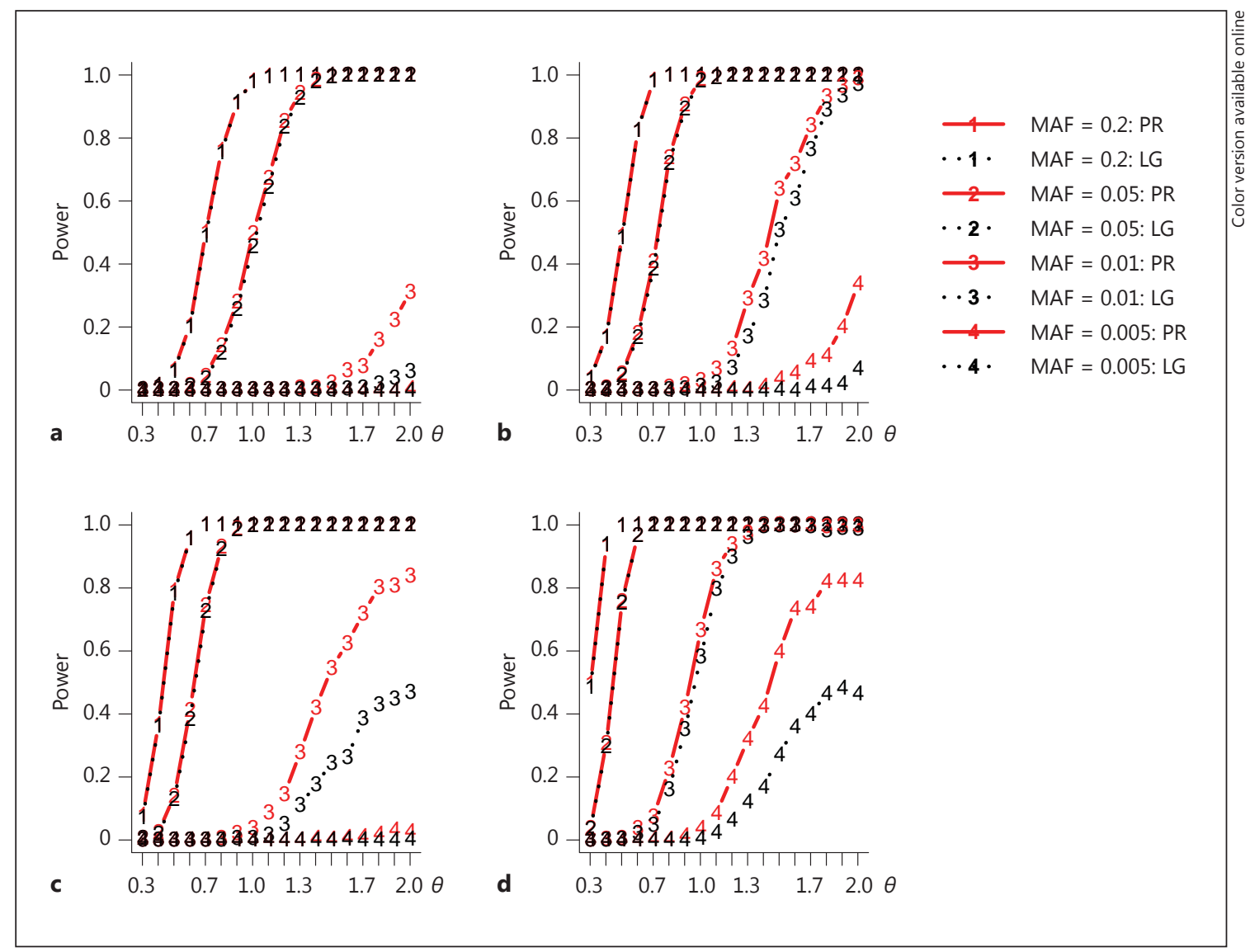

Fig. 2. Power of the Probit (PR) method and the LG method for the dominant model using LGsimu (a, b) and PRsimu (c, d). The significance level of the test was $1 \times 10^{-6} \cdot n=500(\mathbf{a}, \mathbf{c}) . n=1,000(\mathbf{b}, \mathbf{d})$.

method, regardless of the phenotype simulation model, sample size, and disease model. For a rare SNP with a MAF of 0.01 or 0.005 and an effect size of 1.6 or 2 , respectively, the power of the Probit method was generally greater than that of the LG method, regardless of the phenotype simulation model and disease model. The power difference became larger with moderate sample sizes. If the phenotype was simulated using LGsimu under the additive model, with a total sample size of 2,100, the power of the Probit method was $80 \%$, whereas that of the LG method was $73 \%$ for detecting a rare SNP with a MAF of 0.01 and an effect size of 1.6. For a total sample size of 2,700 , the power of the Probit method was $81 \%$, but that of the LG method was only $56 \%$ for detecting a rare SNP with a MAF of 0.005 and an effect size of 2 (fig. 3a). If the phenotype was simulated using PRsimu under the additive model, with a total sample size of 1,000 , the power of the Probit method was $65 \%$, whereas that of the LG method was 30\% for detecting a rare SNP with a MAF of 0.01 and an effect size of 1.6. For a total sample size of 2,000, the power of the Probit method was $83 \%$, whereas that of the LG method was only $48 \%$ for detecting a rare SNP with a MAF of 0.005 and an effect size of 2 (fig. 3b).

The relationship between all parameter values and the ratio of the power of the Probit method to that of the LG method was quantified by simulation studies. The relative power for a wide range of parameter setups $\left(\theta(\neq 0), n, p_{\mathrm{A}}\right.$, and PRsimu or LGsimu) was obtained first, and linear regression analysis was then performed using the log-relative power as the outcome variable and the true parameter values as explanatory variables. The estimated mean log-relative power of Probit to LG for testing $H_{0}: \theta=0$ was $2.90-0.85 \log _{10}(n)-2.47 p_{\mathrm{A}}+0.13 \theta-0.11 I$ (PRsimu). This indicates that the magnitude of $\theta$, sample size $n$, and the MAF of SNP $p_{\text {A }}$ play a dominant role in the relative power for testing $H_{0}: \theta=0$.

Next, we study the performance of the Probit method compared to the entropy-based method [11] by simula- 


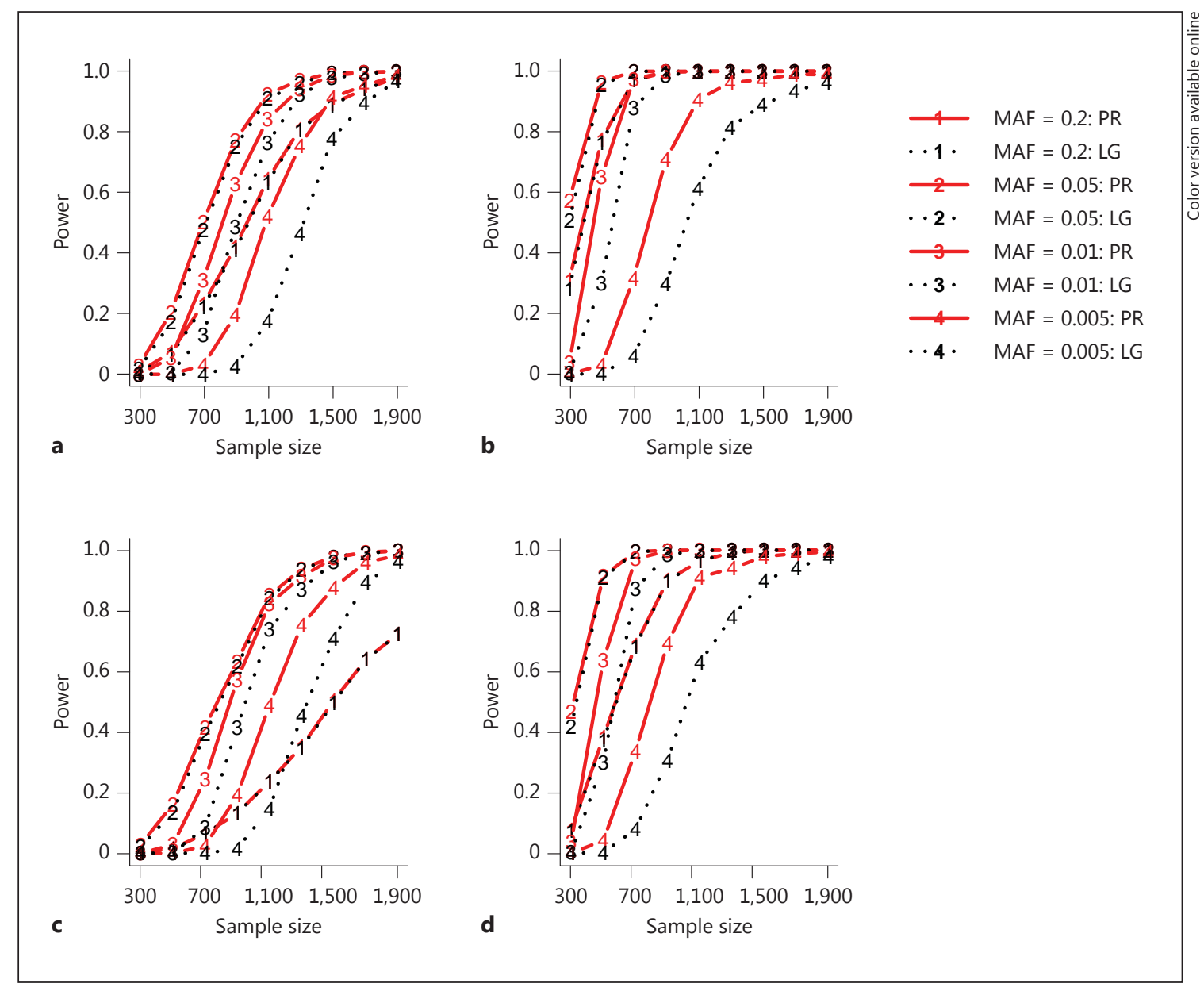

Fig. 3. Power of the Probit (PR) method and the LG method for the additive (a, b) and dominant (c, d) models as a function of sample size using LGsimu (a, c) and PRsimu (b, d), respectively. The significance level of the test was $1 \times 10^{-6}$. The $\beta_{\mathrm{g}}$ values were $0.4,0.8,1.6$, and 2 for SNPs with MAFs of $0.2,0.05,0.01$, and 0.005 , respectively.

tions. Parameter setups were the same as those for figures 1 and 2, except that we did not include any covariates here and that we used sample sizes of $n=1,000$ and 2,000. Figure 4 displays the power difference between the Probit and the entropy-based method for 3 disease models. The power of the Probit method was greater than that of the entropy-based method for the additive and dominant disease models, if the MAFs of the SNP were $0.2,0.05$, and 0.01 (fig. $4 \mathrm{a}, \mathrm{b})$. If the sample size was 2,000, then the Probit method outperformed the entropy-based method for a SNP with a MAF of 0.005 . The maximum of the absolute values of the power differences between the Probit and the entropy-based method for the additive and dominant models was 0.1 . However, for the recessive disease model, the entropy-based method was dominant over the Probit method. The power difference between the Probit and the entropy-based method could be as large as 0.80 (fig. 4c). For SNPs with MAFs 0.01 and 0.005 , there is no power difference between the 2 methods for the recessive disease model, because the power of the 2 methods was 0 due to the small sample size (fig. 4c). These results are in fact consistent with the comparisons between the LG and the entropy-based method in the literature $[11,14]$.

\section{Variance of the Genetic Association Parameter}

\section{Estimates}

To show how the MAF of a SNP, sample size, genetic disease model, and different distribution of noise affect the parameter estimates of Probit and LG, respectively, and how they affect the ultimate power or type I error rate of both methods, respectively, we have conducted a series of simulations with a small sample size, $n=500$, and large 


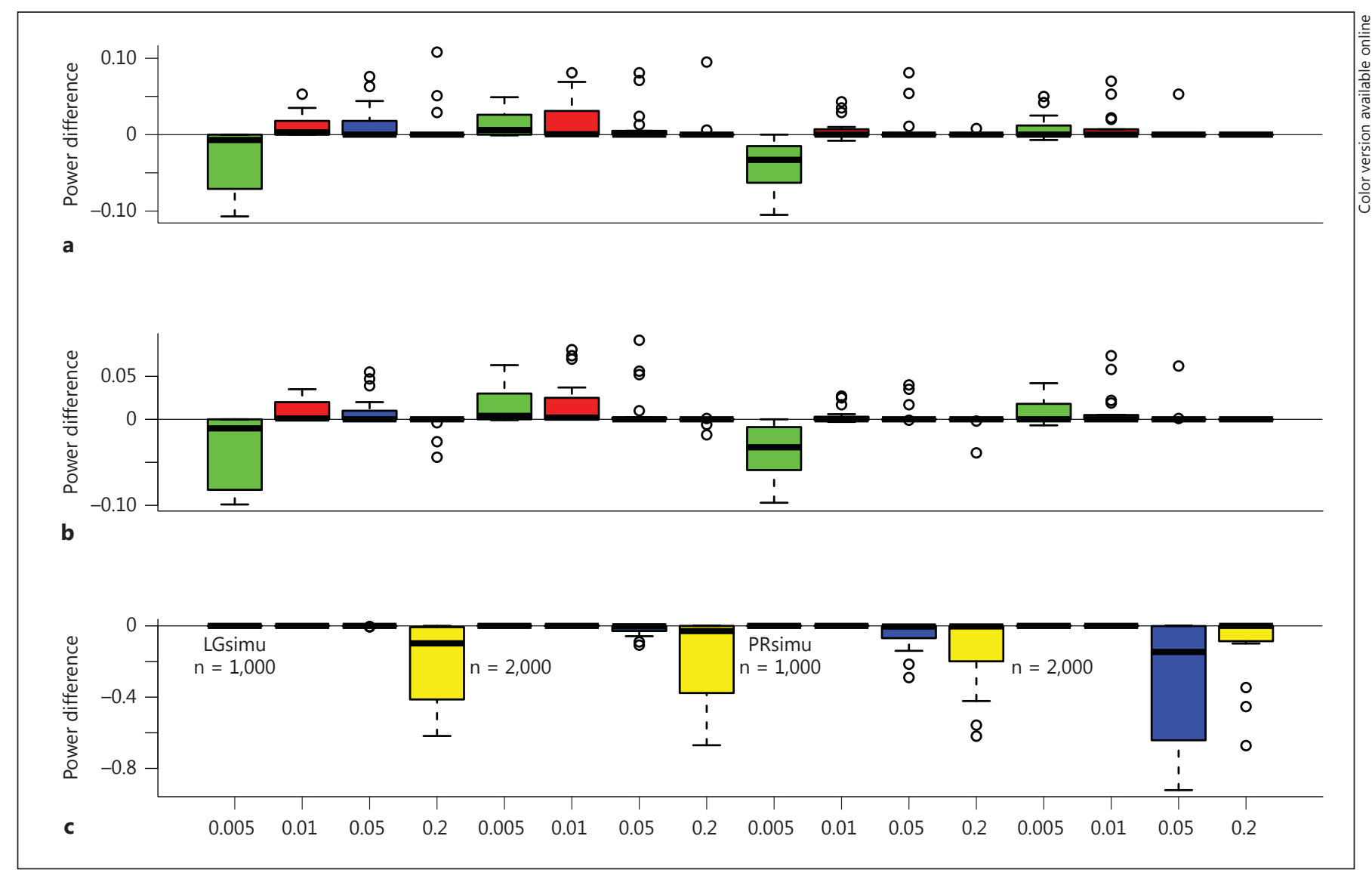

Fig. 4. Power difference between the Probit method and the entropy-based method for the additive (a), dominant (b), and recessive (c) disease models. The first and second four columns were obtained using LGsimu, $n=1,000$ and $n=2,000$, respectively; the third and the last four columns were obtained using PRsimu, $n=$ 1,000 and $n=2,000$, respectively. All parameter setups were the same as in figures 1 and 2 . sample sizes, $n=2,000,3,000,5,000$, and 10,000 , to investigate the genetic association parameter estimates and variance of genetic association parameter estimates.

Table 2 and online supplementary figure $S 1$ show the mean estimates of the genetic effect size association parameter, averaged estimated asymptotic variances, and empirical variances for the LG and Probit methods. Data were generated using the same parameter setup as given in table 1 and figures 1 and 2, except for the sample sizes. If $\theta=0$, regardless of the phenotype simulation model, the mean estimates with both the LG and Probit methods were close to 0 , but estimates with the Probit method were closer to 0 than those with the LG method (table 2, online suppl. fig. S1B). If $\theta \neq 0$, the mean estimates with the LG method appeared to be much closer to the true parameter values than those with Probit if the phenotype was generated from the LG model using LGsimu (and vice versa using PRsimu; table 2, online suppl. fig. S1B). On average, the parameter estimates by the LG method were 2.11 times greater than those by the Probit method for a small sample size of $n=500$, but this factor decreased to 1.83 , $1.80,1.77$, and 1.74 for large sample sizes of $n=2,000$, $3,000,5,000$, and 10,000 , respectively (table 2 , online suppl. fig. S1A). As expected, with the increase of sample size, the estimates become more robust and closer to their true parameter values, regardless of the estimation method (table 2, online suppl. fig. S1A, B). Not surprisingly, the more common the SNP and/or the larger the sample size, the smaller was the bias of its estimate.

The averaged estimated asymptotic variance for the parameter estimates appeared to be close to its empirical counterpart for both the LG and Probit methods for the common SNP (table 2, online suppl. fig. S1C, D). Interestingly, for a small sample size, the averaged estimated as- 
ymptotic variance for the LG method was much larger than its empirical counterpart (mean: 26.9, range: 0.7470.6), especially for rare SNPs (mean: 90, range: 7.6470.6), but not for the Probit method (mean: 1.2, range: 0.6-2.9), regardless of the phenotype simulation model (table 2, online suppl. fig. S1C, D). The association parameter estimates for the Probit method were 2.66-46.8fold less variable than those for the LG method. On average, for a SNP with a MAF of 0.2, the empirical variance obtained by the LG method was 3.48 times (range: $2.67-$ 38) that obtained by the Probit method, which is close to the ratio of the variance of the logistic distribution with a scale of 1 , i.e. $\pi^{2} / 3$, to that of the standard normal distribution, i.e. 1. However, this value increased to 4.94 for a small sample size of $n=500$ (table 2, online suppl. fig. S1F). Interestingly, for a SNP with a MAF of 0.005 , the empirical variance obtained by the LG method was 24.3 times (range: $2.67-195$ ) that obtained by the Probit method, which is about 7 times larger than that for a SNP with a MAF of 0.2. This value increased to 35.1 for a small sample size of $\mathrm{n}=500$ (table 2, online suppl. fig. S1F). All of these simulation results obviously demonstrate that Probit can give more robust and much less variable parameter estimates than LG, it is especially dominant for small sample sizes and rare variants, which translate to a higher power of Probit than that of LG.

\section{Application to the Mini-Exome Data of Genetic Analysis Workshop 17}

To evaluate the performance of the Probit method coupled with the new proposed algorithm, we analyzed data from the Genetic Analysis Workshop 17 (GAW17) which contained 'mini-exome' sequence genotype data of 24,487 SNPs in 3,205 genomic regions of 697 unrelated individuals provided by the 1000 Genome Project [27]. Three quantitative phenotypes were simulated from the normal distribution. Two quantitative phenotypes and one latent disease liability were influenced by 39 SNPs in 9 genes, 72 SNPs in 13 genes, and 51 SNPs in 15 genes, respectively. The third quantitative phenotype was influenced only by the environments and not by genetic variants. One qualitative phenotype, denoted by $Q_{4}$, was simulated based on the 3 quantitative phenotypes and the latent liability, and the top $30 \%$ of the distribution was declared affected. Furthermore, 200 replicate data sets were generated for each phenotype, using fixed genotype data. First, quality control analysis was performed on the SNPs, and SNPs with MAFs $<0.00075$ or HWE test $p$ val-

The Probit Regression to Test Genotype/ Binary Phenotype Associations
Table 3. SNPs associated with $Q_{4}$ for GAW 17 data with p values $<1 \times 10^{-4}$

\begin{tabular}{lllll}
\hline Phenotype dataset & SNP & MAF & Probit & LG \\
\hline 1st & C13S523 & 0.067 & $6.68 \times 10^{-6}$ & $6.57 \times 10^{-6}$ \\
100th & C13S523 & 0.067 & $2.10 \times 10^{-6}$ & $1.98 \times 10^{-6}$ \\
200th & C13S522 & 0.03 & $6.16 \times 10^{-5}$ & $6.52 \times 10^{-5}$ \\
& C13S523 & 0.067 & $7.13 \times 10^{-5}$ & 0.00011 \\
\hline
\end{tabular}

ues $<0.00001$ were excluded. The 1 st, 10 th, 100th, and 200th qualitative traits were used as our outcomes and included age, gender, and smoking status as covariates in both the LG and Probit models.

At a significance level of 0.0001 , no SNP was statistically significant for both the LG and the Probit method for the 10th replicate data. Both methods identified the same causal SNP (C13S523) for the 1st and 100th replicate data. The Probit method identified 2 causal SNPs (C13S523 and C13S522) but the LG method only identified the causal SNP C13S522 for the 200th replicate data (table 3). Neither method identified no-causal SNPs at the significance level of 0.0001 . On average, the Probit method identified fewer non-causal SNPs than but the same number of causal SNPs associated with the qualitative phenotype as the LG method at the significance levels of $0.05,0.01$, and 0.001 (online suppl. table S1).

\section{Application to the ARID5B Gene in Acute Lymphoblastic Leukemia}

Acute lymphoblastic leukemia (ALL) is the most common type of cancer in children and has different incidence rates in different racial/ethnic groups [28]. Genetic variants in ARID5B associated with a risk of ALL have been reported recently $[29,30]$. We analyzed ARID $5 B$ genetic polymorphisms in childhood ALL in two populations of white and Hispanic children [31]. 978 white and 330 Hispanic children enrolled in the Children's Oncology Group clinical trials [32], 1,046 white controls from the Genetic Association Information Network schizophrenia cohort $[33,34]$, and 541 Hispanic controls from HapMap II, the Human Variation Panel, and Mexican participants in the Genetics of Asthma in Latino Americans study [35] were genotyped using Affymetrix SNP Array 6.

After quality control analysis, 49 SNPs within $10 \mathrm{~kb}$ upstream or downstream of the gene were included for 
Table 4. SNPs associated with ALL susceptibility in white and Hispanic children

\begin{tabular}{|c|c|c|c|c|c|c|c|c|}
\hline \multirow[t]{2}{*}{ SNP } & \multicolumn{4}{|c|}{ White children } & \multicolumn{4}{|c|}{ Hispanic children } \\
\hline & MA & MAF & LG & Probit & MA & MAF & LG & Probit \\
\hline rs10821936 & $\mathrm{C}$ & 0.40 & $8.34 \times 10^{-20}$ & $2.70 \times 10^{-20}$ & $\mathrm{~T}$ & 0.43 & $1.03 \times 10^{-7}$ & $6.99 \times 10^{-8}$ \\
\hline rs10821938 & A & 0.48 & $1.47 \times 10^{-14}$ & $8.89 \times 10^{-15}$ & $\mathrm{C}$ & 0.37 & $4.27 \times 10^{-7}$ & $2.74 \times 10^{-7}$ \\
\hline rs10994982 & G & 0.47 & $2.66 \times 10^{-7}$ & $2.41 \times 10^{-7}$ & G & 0.34 & $3.81 \times 10^{-6}$ & $3.47 \times 10^{-6}$ \\
\hline rs7087125 & $\mathrm{T}$ & 0.5 & $9.22 \times 10^{-6}$ & $8.76 \times 10^{-6}$ & $\mathrm{~T}$ & 0.45 & 0.75 & 0.75 \\
\hline rs7896246 & A & 0.40 & $1.03 \times 10^{-19}$ & $3.32 \times 10^{-20}$ & G & 0.45 & $2.77 \times 10^{-7}$ & $2.24 \times 10^{-7}$ \\
\hline rs7923074 & A & 0.48 & $1.50 \times 10^{-13}$ & $9.85 \times 10^{-14}$ & $\mathrm{C}$ & 0.37 & $2.09 \times 10^{-7}$ & $1.35 \times 10^{-7}$ \\
\hline rs2893881 & G & 0.16 & 0.001 & 0.000996 & G & 0.32 & 0.007 & 0.006 \\
\hline
\end{tabular}

MA $=$ Minor allele

association testing of SNP with ALL susceptibility. Table 4 shows the SNPs with p values $<0.001(0.05 / 49)$. We can see that in whites based on the LG method, 6 of the 49 SNPs (rs10821936, rs10821938, rs10994982, rs7087125, rs7896246, and rs7923074) had the $p$ values $<0.001$, and rs 2893881 had a $p$ value of 0.001024 , close to 0.001 . Based on the Probit method, all of these 7 SNPs had $\mathrm{p}$ values $<0.001$ (table 4). In Hispanics, both methods identified the same set of SNPs associated with ALL susceptibility (table 4). At more liberal significance levels of 0.05 and 0.01 , both methods identified the same set of SNPs, too (data not shown).

\section{Discussion}

With the availability of data from whole-genome sequencing and whole-exome sequencing studies, in which moderate sample sizes are used due to the high cost of sequencing technology $[36,37]$, or of data from rare diseases in cancer genomics studies, such as pediatric cancers of retinoblastoma and Ewing's sarcoma [38, 39], there is an increasing demand for the development of powerful and robust association testing procedures for identifying genetic variations associated with a binary phenotype of interest. In this study, we propose a new SV system model, which is a generalized form of the LG and Probit methods, to reproduce the relationship between a binary phenotype and genetic variants and a novel setvalued system identification approach for the Probit method to identifying the parameters' association of interest. We compared it with the LG model. Simulations and real data applications show that the power gain of the Probit compared to the LG method for binary phenotypes is robust to the distributions of noise: logistic or normal distribution, and various genetic disease models, and that the Probit method generally outperforms the commonly used LG method. Furthermore, we also compared the elapsed time between our new algorithm and the built-in command glmfit ( $x, y$, 'binomial', 'link', 'probit') in Matlab using simulations. We found that, on average, the elapsed time of our new algorithm took $0.06 \mathrm{~s}$ (range: 0.0030.1022 ) less than glmfit for one SNP. For GWAS and NGS studies, we usually test for approximately $10^{6}$ and $4 \times 10^{7}$ SNPs, respectively; the new algorithm will save roughly 17 and $677 \mathrm{~h}$, respectively, compared to glmfit in Matlab [40]. However, we did not find a difference of computing time between our new algorithm and the $\mathrm{glm}$ function in R. In addition, the Probit method has greater power than the entropy-based method for the additive and dominant but not for recessive disease models. However, to the best of our knowledge, there is no program available in the entropy-based method to include covariates in genetic association studies. In conclusion, we recommend the use of the Probit method coupled with our new algorithm instead of the LG method, regardless of the distribution of noise, sample size, and effect size of associations between variants and disease of interest, to identify genetic variants, especially rare variants, in genetic association studies.

When we estimate the parameters using a system identification method, we suppose that the variance of noise is known as 1, because we are interested in testing genotype-phenotype associations and not in estimating the effect size of association. In real data analysis, the true variance of noise is usually unknown and it may also not be equal to 1, which will definitely affect the power of the LG and Probit methods. By simulations with noise following 
a normal distribution of $N\left(\mu, \sigma^{2}\right)$, where $\sigma^{2}=3$ and $\sigma^{2}=$ $1 / 3$, not surprisingly, as the true variance of the noise is $>1$, the power of both methods will decrease (and increase when the true variance of the noise is $<1$ ). However, as expected, the power of the Probit method is still identical to or greater than that of the LG method (data not shown). Thus, conclusions about the relative performance of the Probit and LG methods in this study are also robust to the $t$ distribution of the underlying noise. In addition, if we are interested in estimating the association effect size of SNPs on the phenotype, the parameter of variance of noise can also be estimated along with other parameters using an expectation-conditional maximization algorithm [21].

Besides SNP-based analysis, the Probit model coupled with the new algorithm can also be applied to any biologically meaningful mutant and mutant set. It can be applied to a multi-allelic locus and the somatic status of structural variants such as copy number variants, copyneutral regions of loss of heterogeneity, inversions, and translocations. For NGS studies that involve rare variants, due to a lack of power for a single-locus approach, the Probit method can be extended to a multiple locus such as a haplotype-based, gene/set-based, and pathway-based approach for detecting rare variants. Furthermore, the proposed SV model focuses on a binary phenotype with one threshold. However, in real data analyses, especially in the field of pharmacogenomics, the outcome could be multiple ordinal categories such as dosing of drugs, adverse events scored on scales using ordinal values (1-5) according to the Common Terminology Criteria for Adverse Events developed by the National Cancer Institute, or the effect of treatment on disease (e.g. tumor response in which the change of tumor size is categorized as a complete response, partial response, stable disease, or progressive disease) [39]. The concept of a multiple-inputmultiple-output linear system with quantized outputs
[21] can be applied in these cases and hence can provide a comprehensive framework for a wide variety of genetic association studies. Similar to the LG method, the Probit method has also lower power than the entropy-based method if the unknown underlying disease model is recessive. The power of the Probit method can be made robust against the underlying disease models by a computationally intensive approach: as the test statistic, take the maximum of the absolute values of $\mathrm{z}$ test statistics assuming the additive, dominant, and recessive disease models in the Probit model. Then an empirical $p$ value can be obtained by a re-sampling method. In the current study, we have only investigated which method, LG or Probit, performs better in terms of association testing, but in the future, we will determine which method performs better in terms of model fitting and prediction.

We have implemented the Probit model coupled with the proposed EM algorithm in an R package and Matlab codes, which are available for free from http://www. stjuderesearch.org/site/depts/biostats/software. The method can be easily applied to any genetic association study for a binary phenotype, no matter the candidate gene, GWAS or NGS studies.

\section{Acknowledgements}

This research was funded by the American Lebanese and Syrian Associated Charities (ALSAC), the National Natural Science Foundation of China under grants 11171333 and 61134013, NIH grants U01 GM92666 and P30 CA21765, and NCI grants U10 CA98543, U10 CA98413, and U24 CA114766 to the Children's Oncology Group. S.P.H. holds the Ergen Family Chair in Pediatric Cancer. The Genetic Analysis Workshop is supported by the NIH grant R01 GM031575. We thank Dr. Vani Shanker for editing the manuscript and Deqing Pei for providing the ALL data. We thank two anonymous reviewers for their insightful comments which have significantly improved the manuscript.

\section{References}

1 Sladek R, Rocheleau G, Rung J, et al: A genome-wide association study identifies novel risk loci for type 2 diabetes. Nature 2007;445: 881-885.

- 2 The Wellcome Trust Case Control Consortium (WTCCC): Genome-wide association study of 14,000 cases of seven common diseases and 3,000 shared controls. Nature 2007; 447:661-678.

-3 Yang JJ, Cheng C, Devidas M, et al: Genomewide association study identifies germline polymorphisms associated with relapse of childhood acute lymphoblastic leukemia. Blood 2012;120:4197-4204.

4 Bradfield JP, Taal HR, Timpson NJ, et al: A genome-wide association meta-analysis identifies new childhood obesity loci. Nat Genet 2012;44:526-531.

$>5$ Adeyemo A, Gerry N, Chen G, Herbert A, Doumatey A, Huang H, Zhou J, Lashley K, Chen Y, Christman M, Rotimi C: A Genomewide association study of hypertension and blood pressure in African Americans. PLoS Genet 2009;5:e1000564.

6 Florez JC: A genome-wide association study of treated A1C. Diabetes 2010;59:332-334.

7 Armitage P: Tests for linear trends in proportions and frequencies. Biometrics 1955;11: 375-386.

8 Cantor RM, Lange K, Sinsheimer JS: Prioritizing GWAS results: a review of statistical methods and recommendations for their application. Am J Hum Genet 2010;86:6-22.
The Probit Regression to Test Genotype/ Binary Phenotype Associations 
-9 Cochran WG: Some methods for strengthening the common 2 tests. Biometrics 1954;10: 417-451.

10 Sasieni PD: From genotypes to genes: doubling the sample size. Biometrics 1997;53: 1253-1261.

-11 Ruiz-Marín M, Matilla-García M, Cordoba JA, Susillo-González JL, Romo-Astorga A, González-Pérez A, Ruiz A, Gayán J: An entropy test for single-locus genetic association analysis. BMC Genet 2010;11:19.

-12 Freidlin B, Zheng G, Li Z, Gastwirth JL: Trend tests for case-control studies of genetic markers: power, sample size and robustness. Hum Hered 2002;53:146-152 (Erratum in Hum Hered 2009;68:220).

13 Zang Y, Fung WK, Zheng G: Simple algorithms to calculate asymptotic null distributions for robust tests in case-control genetic association studies in R. J Stat Softw 2010; 33:issue 8.

14 Kang G, Zuo Y: Entropy-based joint analysis for two-stage genome-wide association studies. J Hum Genet 2007;52:747-756.

$>15$ Chen J, Chatterjee N: Exploiting HardyWeinberg equilibrium for efficient screening of single SNP associations from case-control studies. Hum Hered 2007;63:196-204.

$>16$ Joo J, Kwak M, Ahn K, Zheng G: A robust genome-wide scan statistic of the Wellcome Trust Case-Control Consortium. Biometrics 2009;65:1115-1122.

$>17$ Zheng G, Ng HKT: Genetic model selection in two-phase analysis for case-control association studies. Biostatistics 2008;9:391399.

18 Joo J, Kwak M, Zheng G: Improving power for testing genetic association in case-control studies by reducing alternative space. Biometrics 2009;66:266-276.

19 Wang L, Zhang J, Yin G: System identification using binary sensors. IEEE Trans Automat Contr 2003;48:1892-1907.
20 Wang L, Yin G, Zhang J, Zhao Y: System Identification with Quantized Observations. Birkhauser, Boston, 2010.

21 Godoy B, Goodwin G, Aguero J, Marelli D, Wigren T: On identification of FIR systems having quantized output data. Automatica 2011;47:1905-1915.

22 Agresti A: Categorical Data Analysis. Hoboken, John Wiley and Sons, 2002.

23 Cakmakyapan S, Goktas A: A comparison of binary logit and probit models with a simulation study. J Soc Econ Stat 2013;2:1-17.

24 Efron B: The efficiency of logistic regression compared to normal discriminant analysis. J Am Stat Assoc 1975;70:892-898.

25 Chambers EA, Cox DR: Discrimination between alternative binary response models. Biometrika 1967;3:573-578.

26 McCullagh P, Nelder J: Generalized Linear Models. London, Chapman and Hall, 1989.

271000 Genomes Project Consortium 2010; Abecasis GR, Altshuler D, et al: A map of human genome variation from population-scale sequencing. Nature 2010;467:1061-1073.

28 Pui CH, Evans WE: Treatment of acute lymphoblastic leukemia. N Engl J Med 2006;354 166-178.

29 Papaemmanuil E, Hosking FJ, Vijayakrishnan J, et al: Loci on 7p12.2, 10q21.2 and $14 \mathrm{q} 11.2$ are associated with risk of childhood acute lymphoblastic leukemia. Nat Genet 2009;41:1006-1010.

30 Treviño LR, Yang W, French D, Hunger SP, Carroll WL, Devidas M, Willman C, Neale G, Downing J, Raimondi SC, Pui CH, Evans WE, Relling MV: Germline genomic variants associated with childhood acute lymphoblastic leukemia. Nat Genet 2009;41:1001-1005.

$31 \mathrm{Xu} \mathrm{H}$, Cheng C, Devidas M, et al: ARID5B genetic polymorphisms contribute to racial disparities in the incidence and treatment outcome of childhood acute lymphoblastic leukemia. J Clin Oncol 2012;30:751-757.
32 Borowitz MJ, Devidas M, Hunger SP, et al: Clinical significance of minimal residual disease in childhood acute lymphoblastic leukemia and its relationship to other prognostic factors: a Children's Oncology Group study. Blood 2008;111:5477-5485.

33 Shi J, Levinson DF, Duan J, et al: Common variants on chromosome $6 \mathrm{p} 22.1$ are associated with schizophrenia. Nature 2009;460: 753-757.

34 International Schizophrenia Consortium; Purcell SM, Wray NR, et al: Common polygenic variation contributes to risk of schizophrenia and bipolar disorder. Nature 2009; 460:748-752.

35 Burchard EG, Avila PC, Nazario S, et al: Lower bronchodilator responsiveness in Puerto Rican than in Mexican subjects with asthma. Am J Respir Crit Care Med 2004;169:386392.

36 Lanktree MB, Hegele RA, Schork NJ, Spence JD: Extremes of unexplained variation as a phenotype: an efficient approach for genomewide association studies of cardiovascular disease. Circ Cardiovasc Genet 2010;3:215-221.

37 Emond MJ, Louie T, Emerson J, et al: Exome sequencing of extreme phenotypes identifies DCTN4 as a modifier of chronic Pseudomonas aeruginosa infection in cystic fibrosis. Nat Genet 2012;44:886-889.

38 Gurney JG, Severson RK, Davis S, Robison LL: Incidence of cancer in children in the United States. Sex-, race-, and 1-year age-specific rates by histologic type. Cancer 1995;75: 2186-2195

39 Wheeler HE, Maitland ML, Dolan ME, Cox NJ, Ratain MJ: Cancer pharmacogenomics: strategies and challenges. Nat Rev Genet 2013; 14:23-34.

40 Bi WJ, Zhao YL: Iterative Parameter Estimate with Batched Binary-Valued Observations: Convergence with an Exponential Rate. Proceedings of the 19th World Congress of the International Federation of Automatic Control, Cape Town, South Africa. 2014. 